\title{
Avaliação da reprodutibilidade ultrassonográfica comométodopara medida do tecido subcutâneo da ponta nasal
}

\section{Evaluation of the ultrasound reproducibility as a method to measure the subcutaneous tissue of the nasal tip}

\author{
Marcell de Melo Naves', Rogério Costa Sousa', Raphael Alves Ferreira Tomé2, Nicholas Godoy Canazza Damian², Angélica \\ Lemos Debs Diniz³, Lucas Gomes Patrocinio4.
}

\author{
1) Médico. Serviço de Otorrinolaringologia da Faculdade de Medicina da Universidade Federal de Uberlândia \\ 2) Médico. Serviço de Radiologia da Faculdade de Medicina da Universidade Federal de Uberlândia. \\ 3) Professor Doutor. Serviço de Radiologia da Faculdade de Medicina da Universidade Federal de Uberlândia. \\ 4) Professor Doutor. Chefe do Serviço de Residência Médica em Otorrinolaringologia da Faculdade de Medicina da Universidade Federal de Uberlândia \\ Instituição: Hospital Santa Genoveva \\ Uberlândia / MG - Brasil. \\ Endereço para correspondência: Lucas Gomes Patrocinio - Rua Artur Bernardes 555, 1² Andar - Uberlândia / MG - Brasil - CEP: $3840-368$ - Telefone: (+55 34) \\ 3215-1143-E-mail: lucaspatrocinio@clinicaotoface.com.br \\ Artigo recebido em 28 de Abril de 2011. Artigo aprovado em 25 de Junho de 2011
}

\section{RESUMO}

Introdução: A prévia avaliação da anatomia do paciente é fundamental para atingir uma ponta nasal bem definida em rinoplastia. A espessura do tecido subcutâneo é essencial para a harmonia estética dessa região. Técnicas de mensuração dessas estruturas no paciente "in vivo" não estão padronizadas na literatura.

Objetivo: Avaliar a variabilidade interobservador do método ultrassonográfico para medida da espessura do tecido subcutâneo da ponta nasal por de ultrasonografia.

Método: 47 voluntários submeteram-se a ultrasonografia da ponta nasal por dois especialistas em radiologia que utilizaram a mesma técnica de exame com transdutor eletrônico linear na frequência de 5 a $9 \mathrm{MHz}$. Avaliou-se a espessura do tecido subcutâneo através da medida do ponto de maior projeção do domo da cartilagem alar maior até a pele. Estudo prospectivo.

Resultados: As médias das medidas do tecido subcutâneo da ponta nasal obtidas pelos examinadores não apresentaram diferenças estatisticamente significante $(\mathrm{p}=0.5303)$. Na análise da reprodutibilidade entre examinadores encontrou-se coeficiente de correlação interclasse r (Pearson) 0,9333, isto é, uma excelente reprodutibilidade interobservadores.

Conclusão: A ultrassonografia demonstrou-se reprodutível e excelente concordância entre os examinadores para avaliação da espessura do tecido subcutâneo da pele, auxiliando o cirurgião na análise pré-operatória do paciente e na escolha da melhor técnica cirúrgica para cada caso.

Palavras-chave: rinoplastia, cartilagens nasais, ultrassonografia.

\section{SUMMARY}

Introduction: The previous evaluation of the patient's anatomy is crucial to achieve a sharp nasal tip in a rhinoplasty. The thickness of the subcutaneous tissue is indispensable for the esthetical harmony of the area. Techniques to measure such structures on the patient 'in vivo' are not found in literature. Objective: Evaluate the inter-observer inconsistency of the ultrasound method to measure the thickness of the subcutaneous tissue of the nasal tip by way of an ultrasound. Method: 47 volunteers were submitted to a nasal tip ultrasound performed by two radiology specialists, who used the same exam technique with a linear electronic transducer at a frequency between 5 and $9 \mathrm{MHz}$. The thickness of the subcutaneous tissue was evaluated by measuring the point with the highest protrusion of the largest alar cartilage dome towards skin. Prospective study.

Results: The measurement averages of the subcutaneous tissue of the nasal tip achieved by the examiners did not show statistically significant differences ( $p=0.5303)$. When analyzing the reproducibility between examiners, an interclass 'r'correlation coefficient (Pearson) 0.9333, i.e., an excellent inter-observer reproducibility was found.

Conclusion: The ultrasound proved to be reproducible and examiners satisfactorily agree about evaluating the thickness of the subcutaneous tissue of the skin, thus helping the surgeon perform the pre-surgical analysis and find the best surgical technique for each case.

Keywords: rhinoplasty, nasal cartilages, ultrasound. 


\section{INTRODUÇÃO}

A avaliação pré-operatória baseada na anatomia do paciente é extremamente importante para o sucesso da rinoplastia $(1,2)$. O tipo de pele, a espessura do tecido subcutâneo, o suporte da ponta e o tamanho, forma e posicionamento das cartilagens alares maiores são de extrema importância na escolha entre as técnicas disponíveis para alcançar uma definição adequada da ponta nasal $(2,3,4,5)$.

A espessura do tecido subcutâneo nasal é extremamente variável. Em um mesmo nariz, normalmente é mais espesso na ponta e na raiz nasal. Varia também de acordo com a raça, sendo mais espesso nos pacientes afrodescendentes do que nos pacientes brancos (6).

O espaço interdomal é definido como o espaço entre a maior projeção das cartilagens alares maiores, sendo tipicamente ocupado por tecido adiposo (7). A forma das cartilagens alares maiores, especialmente se o domo (cúpula) é largo, pode contribuir para a largura do complexo da ponta (6). É considerado normal o ângulo de divergência interdomal de $30^{\circ} \mathrm{e} \mathrm{o}$ arco domal menor ou igual a $4 \mathrm{~mm}$.

Estudos anatômicos em cadáveres demonstram que pontas nasais mal definidas, em caixote e bulbosas apresentam tecido subcutâneo nasal mais espesso (8). Entretanto, técnicas de mensuração da espessura do tecido subcutâneo no paciente "in vivo" não está padronizada na literatura.

O objetivo deste estudo é avaliar a variabilidade interobservador do método ultrassonográfico para medida da espessura do tecido subcutâneo da ponta nasal por meio de técnica padronizada.

\section{MÉTODO}

Foram selecionados 47 voluntários para serem submetidos a ultrassonografia da ponta nasal. Os critérios de inclusão foram: idade entre 18-70 anos. Os critérios de exclusão foram: cirurgia nasal prévia, cicatriz na ponta nasal, trauma nasal prévio e presença de qualquer doença que altere a anatomia da pele, subcutâneo e cartilagens da ponta nasal.

Os exames ultrassonográficos foram realizados por dois médicos especialistas em radiologia, que utilizaram a mesma técnica de exame previamente padronizada. A avaliação de cada paciente foi realizada no mesmo dia, porém os observadores não tiveram acesso aos resultados das medidas anteriormente obtidas, com o objetivo de evitar a contaminação das amostras.

O equipamento utilizado para realização da ultrassonografia foi da marca Medison ${ }^{\circledR}$, modelo Sonoace 8000 SE, com transdutor eletrônico linear na frequência de 5 a 9 MHz. Todos os pacientes foram avaliados em decúbito dorsal. O transdutor foi posicionado sobre a ponta nasal, sem exercer nenhuma pressão sobre a mesma, a fim de não subestimar a aferição. Foi avaliada a espessura do tecido subcutâneo através da medida do ponto de maior projeção do domo da cartilagem alar maior até a pele, quantificada em milímetros.

\section{Análise Estatística}

A análise estatística descritiva foi usada para a caracterização sócio-demográfica e para calcular as médias e desvios-padrão das medidas obtidas das aferições dos dois observadores. Por meio do teste de Lilliefors verificouse a normalidade dos dados. O teste $t$ de Student foi utilizado na comparação das medidas obtidas pelos dois observadores. O coeficiente de correlação intraclasse e o respectivo intervalo de confiança foram utilizados para determinar a reprodutibilidade das medidas obtidas pelos dois examinadores. Utilizou-se também o gráfico de BlandAltman para avaliar a concordância entre medições. O nível de significância estatística foi definido $\operatorname{com} p<0,05$.

\section{RESULTADOS}

O grupo de estudo foi constituído por 47 pacientes, sendo 16 do sexo masculino e 31 do feminino, com idade variando de 18 a 70 anos (média de 35 anos \pm 14 anos).

A medida ultrassonográfica do tecido subcutâneo da ponta nasal foi realizada em média em 1,2 minutos (0,9-2 minutos). As médias das medidas do tecido subcutâneo da ponta nasal obtidas, com seus respectivos desvios-padrão, foram: 4,352 $\mathrm{mm} \pm 0,6222$ para o primeiro examinador $\mathrm{e}$ $4,330 \mathrm{~mm} \pm 0,674$ para o segundo examinador.

As comparações das medidas obtidas pelos avaliadores um e dois não apresentaram diferenças estatisticamente significante ( $p=0.5303)$, indicando que os examinadores não diferem entre si em relação às medidas (Gráfico 1). A boa concordância pode ser confirmada observando-se gráfico das médias. A média das diferenças foi muito baixa, ou seja, $0,02234 \mathrm{~mm}$ e desvio-padrão de $0,24 \mathrm{~mm}$, mostrando excelente concordância entre os examinadores.

$\mathrm{Na}$ análise da reprodutibilidade entre examinadores encontrou-se coeficiente de correlação interclasse 1 


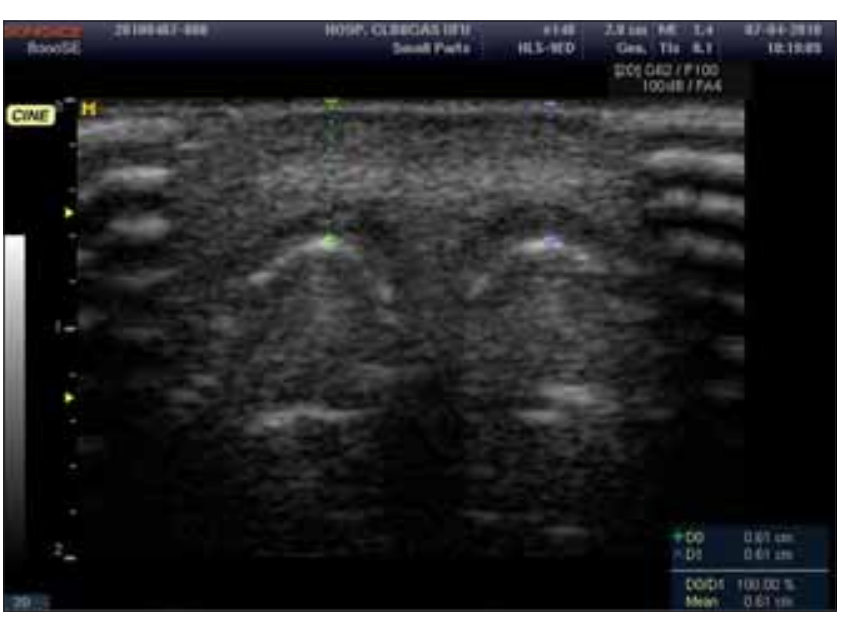

Figura 1. Imagem ultrasonográfica da ponta nasal evidenciando a medida da espessura do tecido subcutâneo da ponta nasal (maior projeção do domo da cartilagem alar maior até a pele).

(Pearson) 0,9333 (intervalo de confiança [IC] 95 $=0,88$ a $0,96 p<0.0001$ ), isto é, uma excelente reprodutibilidade interobservadores.

\section{DISCUSSÃO}

A cirurgia da ponta nasal é uma parte extremamente complexa da rinoplastia (1). Como esta, é influenciada por fatores como tipo de pele, espessura do tecido subcutâneo, suporte da ponta e tamanho, forma e posicionamento das cartilagens alares maiores. Portanto, o conhecimento anatômico detalhado dessa região auxilia o cirurgião no planejamento cirúrgico e decisão da técnica a ser aplicada, influenciando diretamente no resultado estético final $(2,3,4)$.

Ademais, a espessura da pele e do tecido subcutâneo é um fator extremamente importante para a escolha da técnica a ser usada para alcançar uma melhor definição da ponta nasal (5). Logo, a análise ultrassonográfica da ponta nasal concomitantemente à análise pré-operatória pode auxiliar o cirurgião a decidir sobre a técnica a ser escolhida para cada paciente.

A utilização da ultrassonografia na avaliação da ponta nasal foi introduzida por TASman e Helbig, em 2000, com o objetivo de avaliar as modificações ocorridas na ponta nasal em decorrência da rinoplastia (9). Em 2004, Copcu et al. aplicaram a ultrassonografia para demonstrar a presença do tecido adiposo interdomal como uma estrutura independente ocupando o espaço interdomal na ponta nasal (8). Coskun et al., em 2008, publicaram a análise tridimensional do tecido adiposo interdomal através da ultrassonografia com medidas vertical, transversa e longitu-

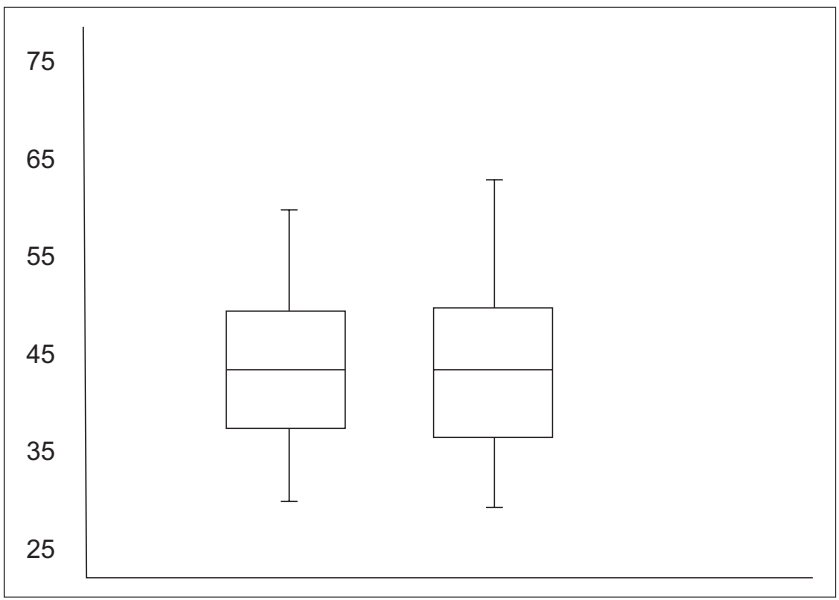

Gráfico 1. Médias e desvios-padrão das medidas obtidas pelos examinadores 1 e 2 . Não se observam diferenças significativas entre as médias das três medidas obtidas pelos dois examinadores.

dinal dessa estrutura e a confirmação da mesma pela dissecção (10). Entretanto, nenhum estudo foi realizado para validação do método para avaliação da espessura do tecido cutâneo da ponta nasal.

A definição da ponta nasal pode ser atingida por vários métodos, incluindo técnicas de sutura, ressecções e enxertos $(11,12)$. A escolha da técnica a ser aplicada em cada paciente é totalmente dependente da pele e do tecido subcutâneo. As técnicas de sutura são aplicadas para pacientes de pele de espessura fina e normal e objetivam a correção de deformidades das cartilagens da ponta nasal naqueles pacientes com ponta em caixote $(5,13,14,15)$ Pacientes com deformidades maiores da ponta nasal e pele de espessura grossa não são satisfatoriamente abordados com técnicas de sutura. Necessitam de enxertos para promover definição e refinamento adequados da ponta nasal (16). Técnicas de divisão de domo também podem ser necessárias em pacientes de pele extremamente espessa $(17,18)$.

Vários autores concordam que a melhora da definição da ponta nasal deve ter como princípio à aplicação de técnicas mais conservadoras para alterações mais discretas em indivíduos de pele e tecido subcutâneo finos e técnicas mais agressivas para deformidades maiores em pacientes de pele e tecido subcutâneo espessos (5).

A avaliação ultrassonográfica da ponta nasal é um método objetivo de identificação da anatomia dessa região e mostrou-se eficiente na avaliação da pele e tecido subcutâneo da ponta nasal. Os resultados obtidos evidenciaram boa reprodutibilidade sem diferença estatisticamente significante nas medidas obtidas pelos examinadores, fato que dá confiabilidade ao método como mais uma 
arma para o cirurgião na análise pré-operatória e consequentemente na programação cirúrgica.

\section{CONCLUSÃO}

A utilização da ultrassonografia para avaliação da espessura do tecido subcutâneo da pele demonstrou-se reprodutível e de excelente concordância entre os examinadores. É um método seguro, não invasivo e de baixo custo que pode auxiliar o cirurgião na avaliação da pele e tecido subcutâneo do paciente e na escolha da melhor técnica cirúrgica para cada caso.

\section{REFERÊNCIAS BIBLIOGRÁFICAS}

1. Ghavami A, Janis JE, Acikel C, Rohrich RJ. Tip shaping in primary rhinoplasty: an algorithmic approach. Plast Reconstr Surg. 2008, 122(4):1229-1241.

2. Toriumi DM. New concepts in nasal tip contouring. Arch Facial Plast Surg. 2006, 8(3):156-185.

3. Tardy MEJr. Graduated sculpture refinement of the nasal tip. Facial Plast Surg Clin North Am. 2004, 12(1):51-80.

4. Patrocínio LG, Carvalho PM, de Souza HM, Couto HG, PatrocínioJA. Surgical maneuvers performed on rhinoplasty procedures carried out at an otorhinolaryngology residency program. Braz J Otorhinolaryngol. 2006, 72(4):439-442.

5. Patrocínio LG, Patrocínio TG, Maniglia JV, Patrocínio JÁ. Graduated Approach to Refinement of the Nasal Lobule. Arch Facial Plast Surg. 2009, 11(4):221-229.

6. Daniel RK. The nasal tip: anatomy and aesthetics. Plast Reconstr Surg. 1992, 89(2):216-224.

7. Sun GK, Lee DS, Glasgold AI. Interdomal fat pad: An important anatomical structure in rhinoplasty. Arch Facial Plast Surg. 2000, 2:260-263.
8. Copcu E, Metin K, Ozsunar Y, Çulhaci N, Ozkök S. The interdomal fat pad of the nose: A new anatomical structure. Surg Radiol Anat. 2004, 26(1):14-18.

9. Tasman, AJ, Helbig M. Sonography of nasal tip anatomy and surgical tip refinement. Plast Reconstr Surg. 2000, 105:2573-2579.

10. Coskun N, Yavuz A, Dikici MB, Sindel T, Islamoglu K, Sindel M. Three-Dimensional Measurements of the Nasal Interdomal Fat Pad. Aesth Plast Surg. 2008, 32:262-265.

11. Crumley RL, Lanser M. Quantitative analysis of nasal tip projection. Laryngoscope. 1998, 202-208

12. Tardy M-E, Cheng E. Transdomal suture refinement of the nasal tip. Facial Plast Surg. 1997, 4:317-320

13. Guyuron B, Behmand RA. Nasal tip sutures part II: the interplays. Plast Reconstr Surg. 2003, 112:1130-45.

14. Behmand RA, Ghavami A, Guyuron B. Nasal tip sutures Part I: the evolution. Plast Reconstr Surg. 2003, 112:11259 .

15. Pedroza F. A 20-year review of the "new domes" technique for refining the drooping nasal tip. Arch Facial Plast Surg. 2002, 4(3):157-163.

16. Pastorek NJ, Bustillo A, Murphy MR, Becker DG. The extended columellar struttip graft. Arch Facial Plast Surg. 2005, 7(3):176-184.

17. Simons RL. Vertical dome division in rhinoplasty. Otolaryngol Clin North Am. 1987, 20(4):785-796.

18. Patrocínio LG, PatrocínioJA. Open rhinoplasty in African American noses. Br J Oral Maxillofac Surg. 2007, 45(7):561566. 